\title{
Using two-stream theory to capture fluctuations of satellite-perceived TOA SW radiances reflected from clouds over ocean
}

\author{
Florian Tornow ${ }^{1,2,3}$, Carlos Domenech ${ }^{4}$, Howard W. Barker ${ }^{5}$, René Preusker ${ }^{1}$, and Jürgen Fischer ${ }^{1}$ \\ ${ }^{1}$ Institute for Space Sciences, Freie Universität Berlin, Berlin, Germany \\ ${ }^{2}$ Center for Climate Systems Research, Earth Institute, Columbia University, New York, NY, USA \\ ${ }^{3}$ NASA Goddard Institute for Space Studies, New York, NY, USA \\ ${ }^{4}$ GMV, Madrid, Spain \\ ${ }^{5}$ Environment and Climate Change Canada, Toronto, Ontario, Canada
}

Correspondence: Florian Tornow (florian.tornow @ fu-berlin.de)

Received: 18 April 2020 - Discussion started: 22 April 2020

Revised: 25 June 2020 - Accepted: 29 June 2020 - Published: 21 July 2020

\begin{abstract}
Shortwave (SW) fluxes estimated from broadband radiometry rely on empirically gathered and hemispherically resolved fields of outgoing top-of-atmosphere (TOA) radiances. This study aims to provide more accurate and precise fields of TOA SW radiances reflected from clouds over ocean by introducing a novel semiphysical model predicting radiances per narrow sun-observer geometry. This model was statistically trained using CERES-measured radiances paired with MODIS-retrieved cloud parameters as well as reanalysis-based geophysical parameters. By using radiative transfer approximations as a framework to ingest the above parameters, the new approach incorporates cloudtop effective radius and above-cloud water vapor in addition to traditionally used cloud optical depth, cloud fraction, cloud phase, and surface wind speed. A two-stream cloud albedo - serving to statistically incorporate cloud optical thickness and cloud-top effective radius - and Cox-Munk ocean reflectance were used to describe an albedo over each CERES footprint. Effective-radius-dependent asymmetry parameters were obtained empirically and separately for each viewing-illumination geometry. A simple equation of radiative transfer, with this albedo and attenuating above-cloud water vapor as inputs, was used in its log-linear form to allow for statistical optimization. We identified the two-stream functional form that minimized radiance residuals calculated against CERES observations and outperformed the state-ofthe-art approach for most observer geometries outside the sun-glint and solar zenith angles between 20 and $70^{\circ}$, reduc-
\end{abstract}

ing the median $\mathrm{SD}$ of radiance residuals per solar geometry by up to $13.2 \%$ for liquid clouds, $1.9 \%$ for ice clouds, and $35.8 \%$ for footprints containing both cloud phases. Geometries affected by sun glint (constituting between $10 \%$ and $1 \%$ of the discretized upward hemisphere for solar zenith angles of 20 and $70^{\circ}$, respectively), however, often showed weaker performance when handled with the new approach and had increased residuals by as much as $60 \%$ compared to the state-of-the-art approach. Overall, uncertainties were reduced for liquid-phase and mixed-phase footprints by $5.76 \%$ and $10.81 \%$, respectively, while uncertainties for ice-phase footprints increased by $0.34 \%$. Tested for a variety of scenes, we further demonstrated the plausibility of scene-wise predicted radiance fields. This new approach may prove useful when employed in angular distribution models and may result in improved flux estimates, in particular dealing with clouds characterized by small or large droplet/crystal sizes.

\section{Introduction}

Radiative fluxes at the top of the atmosphere (TOA) inferred from satellite observations serve many purposes. Instantaneous flux estimates paired with properties of underlying clouds, aerosols, atmospheric gases, and Earth's surface may inform us about the radiative effect of each component of Earth's radiation budget (e.g., Loeb and Manalo- 
Smith, 2005; Li et al., 2011; Thorsen et al., 2018). TOA fluxes may also help to constrain uncertainties concerning cloud-aerosol-radiation interactions, which will be tested in the EarthCARE satellite mission (Illingworth et al., 2015). In EarthCARE's radiative closure assessment, observationbased fluxes will be used to help continuously assess both active-passive retrievals of cloud and aerosol properties and results from radiative transfer simulations performed on them (Barker et al., 2011; Barker and Wehr, 2012). Integrals of estimated fluxes over large areas and long time spans (Loeb et al., 2018) help us understand the Earth-atmosphere system's current radiation budget (e.g., Stephens et al., 2012), thus helping to verify global climate models (e.g., Bender et al., 2006; Boucher et al., 2013; Calisto et al., 2014; Nam et al., 2012).

Inferring fluxes from satellite-based radiometry involves a number of steps. The key challenge for solar fluxes, the general focus of this paper, is that constituents such as clouds reflect solar radiation unevenly across the upward hemisphere, and we need to assume how measurements from a subset of directions relate to radiances in directions not viewed. The intention is to adequately represent hemispheric distributions of radiances such that when integrated yield accurate flux estimates. The solution to this challenge has been empirical angular distribution models (ADMs) that learn, via statistical approaches, hemispherically resolved radiance fields associated with atmospheric scenes using years of satellite observations. For clouds over ocean, the specific concern of this paper, early efforts (Suttles et al., 1988; Smith et al., 1986) worked with ERBE (Earth Radiation Budget Experiment) radiometry as well as GOES (Geostationary Operational Environmental Satellite) measurements and defined four scene types ranging in cloud coverage (including "clear ocean", which used a cloud cover up to $5 \%$; two cloudy scene types over ocean; and "overcast", which blended all surface types). Observations were sorted to produce mean radiances per observed angular ranges for each illumination geometry. Using CERES (Clouds and the Earth's Radiant Energy System) and VIRS (Visible and Infrared Scanner) on the TRMM (Tropical Rainfall Measuring Mission) satellite, Loeb et al. (2003) refined this method and sorted observations into combinations of 12 cloud coverage classes and 14 cloud optical thickness groups and treated ice- and liquid-phase clouds separately. Instead of a discrete scenetype definition, Loeb et al. (2005) defined a continuous description of scene type for the Terra mission, using a sigmoidal function to fit cloud optical thickness and cloud fraction based on MODIS (Moderate Resolution Imaging Spectroradiometer) measurements with CERES-measured TOA shortwave (SW) radiances. They treated footprints containing both ice- and liquid-phase clouds (throughout the paper referred to as "mixed phase") separately from pure and ice and liquid cases. Much of their state-of-the-art methodology was adapted for the Aqua mission (also hosting CERES and
MODIS instruments) using improved cloud algorithms and longer data records (Su et al., 2015).

A recent case study (Tornow et al., 2018) focused on marine stratocumulus-like clouds of optical thickness $\tilde{\tau} \approx 10$ and identified additional parameters that influence ADMs: above-cloud water vapor (ACWV) and layer mean cloudtop effective radius $\overline{R_{\mathrm{e}}}$. They showed that ignoring these parameters could cause deviations in instantaneous flux estimates of about $10 \mathrm{Wm}^{-2}$. This suggests the non-negligible role of solar absorption and single scattering for determination of cloud reflectance patterns. Features of single scattering, such as the cloud bow and glory for liquid clouds or the specular reflection peak for ice clouds, were generally visible in earlier ADMs (e.g., Loeb et al., 2005). These features - solely shaped by the particle phase function that largely depends on particle shape and size - can occur for a wide range of cloud optical thicknesses. Using simulated radiance fields, Gao et al. (2013) demonstrated that scattering regimes, ranging from foremost single scattering to Lambertian-like multiscattering mediums, are functions of the cloud optical thickness. For an intermediate regime, which showed singlescattering features, Gao et al. speculated that the uppermost $\tau \approx 1$ of cloud is responsible for single-scattering contributions.

This study presents a novel semiphysical model that predicts TOA SW radiances for cloudy scenes over ocean for narrow ranges of sun-observer angles. Estimates are sensitive to $\overline{R_{\mathrm{e}}}$ and ACWV and are compared to results from the stateof-the-art methodology. This new approach used the twostream approximation to statistically ingest MODIS cloud properties and other geophysical auxiliary parameters. We began by finding the framework of approximations that best explained CERES-observed radiance fluctuations and then demonstrated that semiphysical log-linear models produced tenable radiance fields.

Section 2 presents data from Aqua and Terra satellites used in the current study. Section 3 explains both the state-ofthe-art methodology for radiance estimation and the new approach. Section 4 identifies optimal solutions and assesses their properties. Section 5 discusses results and conclusions.

\section{Data}

Measured TOA SW radiances paired with scene properties - including imager-based cloud properties and further geophysical auxiliary parameters - were obtained from the CERES Ed4SSF (Edition 4.0 Single Scanner Footprint) dataset of Aqua and Terra satellite missions, primarily from days during years 2000-2005 when CERES instruments were measuring in rotating azimuth plane scan mode to provide angular coverage for ADM construction.

We extracted parameters concerning CERES broadband radiometry. Apart from upwelling unfiltered TOA SW radiances $I^{*}$, covering the spectral range of $0.4-4.5 \mu \mathrm{m}$, and their 
angular geometry (i.e., solar zenith angle $\theta_{0}$, viewing zenith angle $\theta_{\mathrm{v}}$, and relative azimuth angle $\varphi$ ), we collected downwelling TOA SW fluxes $F^{\downarrow}$ that incorporate each measurements' prevalent Sun-Earth distance, which allowed normalization of gathered radiances via $I=I^{*} \frac{S_{0} \cos \theta_{0}}{F^{\downarrow}}$, with solar constant $S_{0}=1361.0 \mathrm{Wm}^{-2}$.

Collocated to each CERES footprint, the SSF dataset summarizes cloud property retrievals (Sun-Mack et al., 2018) on the MODIS pixel level taking into account the CERES point spread function (PSF) (Wielicki et al., 1996) and reports properties for up to two cloud layers per footprint (given that both layers' cloud-top pressure differed by $50 \mathrm{hPa}$ or more; Loeb et al., 2003). We extracted layer cloud fraction $f$ and several statistics on the retrieved field of cloud optical depth $\tau$ (layer average of its logarithm $\tilde{\tau}=e^{\overline{\log \tau}}$, layer average $\bar{\tau}$, and layer SD $\sigma(\tau)$ ), as well as layer mean values of cloud condensate phase $\phi$ (i.e., liquid, ice, or a mixture of both; involving MODIS band $3.7 \mu \mathrm{m}$ ), effective radii of water or ice particles $\overline{R_{\mathrm{e}}}$ (using band $3.7 \mu \mathrm{m}$ ), and cloud-top pressure $p^{\text {ctop }}$. A quality flag summarizing the retrieval confidence (using the parameter "Note for cloud layer" from the SSF dataset) was also collected.

Additional geophysical auxiliary parameters provided in the SSF dataset were extracted. We obtained a surface broadband albedo $\alpha^{\text {surface }}$, surface IGBP (International GeosphereBiosphere Programme) types, and $10 \mathrm{~m}$ surface wind speed $w_{10 \mathrm{~m}}$. The wind speed parameter stemmed from GEOS data assimilation version 5.4.1.

Lastly, to incorporate above-cloud water vapor (ACWV) into our analysis, we used layer mean cloud-top pressure (of the layer with larger cloud fraction) and extracted from ERA-20C (ECMWF 20th century) reanalysis (Poli et al., 2016) four-dimensional fields whose vertical profiles of relative humidity $\mathrm{RH}(p)$ and temperature $T(p)$ that were nearest in time and geolocation to the footprint center. For each CERES footprint we collocated the following vertical integral of mixing ratio $\operatorname{mr}(p)$, with saturation vapor pressure $e_{\mathrm{S}}=6.112 e^{\frac{17.67 T}{T+243.5}}$ (using $T$ in ${ }^{\circ} \mathrm{C}$ ) (Bolton, 1980), gravitational acceleration $g$, and molecular weights of water and dry air $\mathrm{mol}_{\mathrm{h} 2 \mathrm{o}}$ and $\mathrm{mol}_{\text {air }}$ respectively:

$$
\begin{aligned}
\mathrm{ACWV} & =\frac{1}{g} \int_{p^{\text {ctop }}}^{0} \operatorname{mr}(p, T, \mathrm{RH}) \mathrm{d} p \\
& =\frac{1}{g} \int_{p^{\text {ctop }}}^{0} \frac{e_{\mathrm{s}}(T)}{p} \frac{\operatorname{mol}_{h 20}}{\operatorname{mol}_{\text {air }}} \mathrm{RH}(p) \mathrm{d} p .
\end{aligned}
$$

For footprints consisting of multiple cloud layers, relying on a single cloud-top pressure may introduce uncertainty, especially for mixed-phase footprints (see Sect. 3) where pressure difference between ice- and liquid-phase layers is exceptionally large.
Table 1. Number of CERES footprints obtained after screening for marine clouds. Number are shown in millions (in total 1711937663 footprints).

\begin{tabular}{rrrr}
\hline & \multicolumn{2}{c}{ No. of CERES footprints $\left(\times 10^{6}\right)$} & \\
\cline { 2 - 3 } Year & $\begin{array}{r}\text { Terra } \\
\text { Aqua }\end{array}$ & Mode \\
& $($ FM1 and FM2) & $($ FM3 and FM4) & \\
\hline 2000 & 164.02 & $/$ & RAPS \\
2001 & 228.10 & $/$ & RAPS \\
2002 & 236.74 & 84.39 & RAPS \\
2003 & 236.60 & 203.30 & RAPS \\
2004 & 243.53 & 245.65 & RAPS \\
2005 & 6.05 & 63.56 & RAPS \\
\hline
\end{tabular}

For our analysis, we filtered the extracted dataset for samples with more than $95 \%$ water surface, more than $0.1 \%$ cloud fraction, and solar zenith angles between 0 and $82^{\circ}$. Table 1 lists the resulting subset of 1.7 billion samples.

\section{Methods for capturing radiance fluctuations}

In order to provide hemispherically resolved fields of backscattered radiances to radiance-to-flux-converting ADMs, statistical approaches capture observed radiances together with prevalent scene properties per narrow and discretized sun-observer geometry. Following $\mathrm{Su}$ et al. (2015), solar zenith, viewing zenith, and relative azimuth angles were discretized into $2^{\circ}$ intervals, referred to as $\theta_{0}^{\Delta}$, $\theta_{\mathrm{v}}^{\Delta}$, and $\varphi^{\Delta}$, respectively. Combinations of $\theta_{0}^{\Delta}, \theta_{\mathrm{v}}^{\Delta}$, and $\varphi^{\Delta}$ were denoted as angular bins, and observations were sorted into bins for separate treatment. The following subsection presents the state-of-the-art methodology. Section 3.2 introduces a novel semiphysical approach that includes additional parameters.

\subsection{State-of-the-art approach (Su et al., 2015)}

An analytic sigmoidal function related TOA SW radiance with MODIS-based $f$ and $\tilde{\tau}$.

$I\left(\theta_{0}^{\Delta}, \theta_{\mathrm{v}}^{\Delta}, \varphi^{\Delta}\right)=I_{0}+\frac{a}{\left[1+e^{-\frac{\left(x-x_{0}\right)}{b}}\right]^{c}}$,

where $x=\log f \tilde{\tau}$ for a single cloud layer or $x=\log \left[\left(f_{1}+\right.\right.$ $\left.f_{2}\right) e^{\frac{f_{1} \log \tilde{1}_{1}+f_{2} \log \tilde{\tau}_{2}}{f_{1}+f_{2}}}$ for two layers, and $I_{0}, a, b, c$, and $x_{0}$ were free parameters. Optimization of sigmoidal parameters relied on mean radiances that were produced per $x$ interval (every 0.02, shown as black dots in Fig. 1a).

Models were generated separately per cloud phase. A footprint's cloud phase was determined via an effective phase, defined as $\phi_{\text {eff }}=\frac{f_{1} \phi_{1}+f_{2} \phi_{2}}{f_{1}+f_{2}}$ for two layers, and the following thresholds: liquid for $1<\phi_{\text {eff }}<1.01$, mixed for $1.01 \leq$ $\phi_{\text {eff }} \leq 1.75$, and ice for $1.75<\phi_{\text {eff }} \leq 2$. 

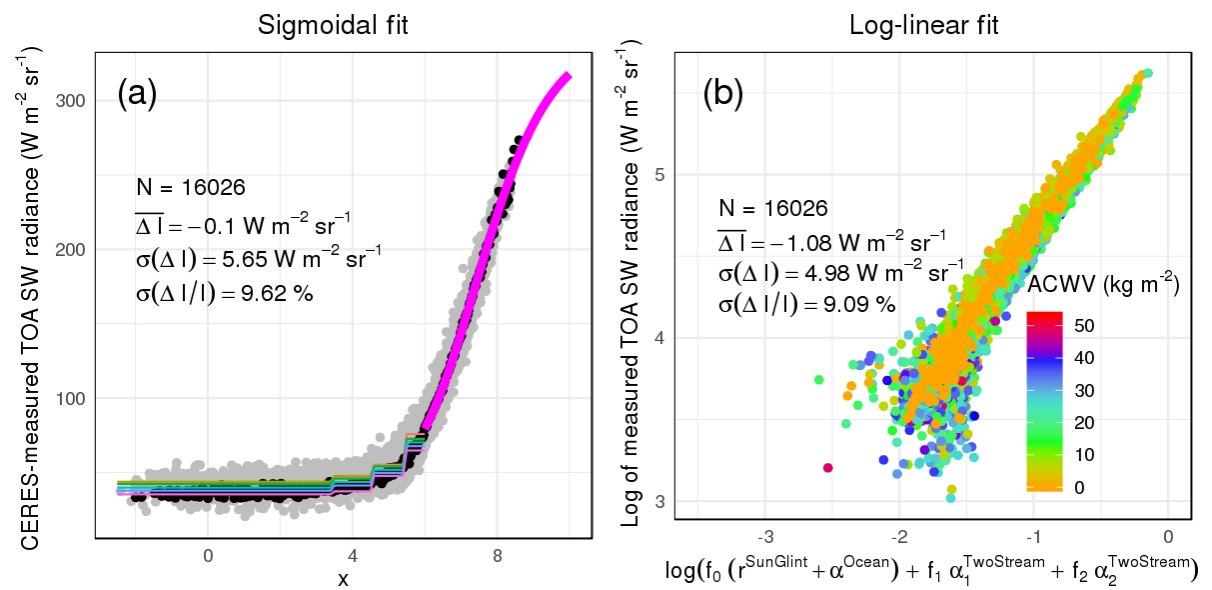

Figure 1. For an example angular bin $\left(\theta_{0} \in\left[20^{\circ}, 22^{\circ}\right], \theta_{\mathrm{v}} \in\left[6^{\circ}, 8^{\circ}\right], \varphi \in\left[12^{\circ}, 14^{\circ}\right]\right)$, we show how a state-of-the-art sigmoidal fit (a) and proposed log-linear model (b) capture fluctuations of CERES-measured TOA SW radiances. As this angular bin is within the sun-glint region, panel (a) shows the lookup-table approach for $x<6$ (as defined in Sect. 3.1; note that $f_{1}$ and $f_{2}$ are taken between 0 and 100 in panel $\mathbf{a}$ and between 0 and 1 in panel $\mathbf{b}$ ). Colors in (b) mark the amount of above-cloud water vapor. Statistics in both panels summarize each approach's number of samples, bias, and SD of radiance residuals as well as relative deviations.

To handle radiance fluctuations caused by sun glint, a glint region was defined (sun-glint angles $<20^{\circ}$ ). Observations with $x>6$ in affected geometries remained captured by a sigmoid fit. For $x \leq 6$, on the other hand, a lookup-table approach stored mean radiances per wind speed interval $(0-$ $2,2-4,4-6,6-8,8-10$, and $>10 \mathrm{~m} \mathrm{~s}^{-1}$ ) and per $x$ interval $(<3.5,3.5-4.5,4.5-5.5,5.5-6)$.

The selected angular bin in Fig. 1 had a sun-glint angle of about $14^{\circ}$ and shows how tabulated radiances (colors correspond to wind speed intervals) and the sigmoidal curve both covered observed radiances.

\subsection{Novel semiphysical approach}

There are several ways one might incorporate additional variables $\overline{R_{\mathrm{e}}}$ and ACWV into a radiance-predicting statistical model. One could divide each angular bin's samples into classes of $\overline{R_{\mathrm{e}}}$ and ACWV and repeat sigmoidal fitting for each combination of classes (see Sect. 3.1). Some bins, however, contained too few samples or failed to cover the full spectrum of at least one of the two parameters. As a viable alternative, we explored radiative transfer approximations as a way to ingest scene properties (i.e., MODIS-based cloud properties and geophysical auxiliary parameters), and this allowed incorporating all samples in a continuous manner.

Working with cloudy atmospheres over ocean surfaces, we assumed that radiance fluctuations were mainly driven by the bidirectional reflection of clouds and water surfaces and by directional absorption through water vapor located above (highly reflective) clouds. We initially set out with the following simple equation of radiative transfer:

$I\left(\theta_{0}^{\Delta}, \theta_{\mathrm{v}}^{\Delta}, \varphi^{\Delta}\right) \approx S_{o} \cos \theta_{0} \alpha e^{-2 \mathrm{ACWV}}$, with solar influx $S_{o} \cos \theta_{0}$ and the albedo $\alpha$ of an Earthatmospheric scene covered by the CERES footprint (hereafter referred to as footprint albedo).

In the following subsection we present how footprint albedo was approximated. This then allowed us to use Eq. (3) in its log-linear form and weight the contribution of reflection and absorption via ordinary least-square fitting with free parameters $A, B$, and $C$.

$\log I\left(\theta_{0}^{\Delta}, \theta_{\mathrm{v}}^{\Delta}, \varphi^{\Delta}\right) \approx A+B \log \alpha+C \mathrm{ACWV}$

Like the state-of-the-art methodology (Sect. 3.1), we applied this approach per angular bin (resolved by $2^{\circ}$ in $\theta_{0}, \theta_{\mathrm{v}}$, and $\varphi$ ), allowing us to treat $S_{o} \cos \theta_{0}$ as constant. We also separated by cloud phase but chose a different threshold to discriminate phase. As elaborated in more detail below, we rely on pure liquid and ice phases to, then, treat the mixed phase. Therefore, we consider a footprint as liquid phase for $\phi_{1 / 2}=1$, as ice for $\phi_{1 / 2}=2$, and as mixed for $\phi_{1}=1$ and $\phi_{2}=2$. $\phi_{1 / 2}$ 's were rounded if their values were neither 1 nor 2 .

\subsubsection{Approximating CERES footprint albedo}

To approximate the albedo within each CERES footprint by means of MODIS-based cloud properties and additional geophysical variables ( $\alpha^{\text {surface }}, w_{10 \mathrm{~m}}$, ACWV), we separately handled clear and cloudy portions.

For clear portions within each footprint, we used the surface broadband albedo of underlying water bodies (referred to as $\alpha^{\text {ocean }}=\alpha^{\text {surface }}$; see Sect. 2). To capture sun glint, i.e., the specular reflection at the ocean's surface that is altered as low-level winds perturb the water surface and tilt reflective facets, we used a Cox-Munk reflectance (Cox and Munk, 
1954), as formulated in Wald and Monget (1983) with Fresnel reflection factor $\rho(\omega)$ for a perfectly smooth surface, and sun-observer geometry per CERES footprint:

$r^{\text {SunGlint }}=\frac{\pi \rho(\omega) P\left(\theta_{n}, W_{10 \mathrm{~m}}\right)}{4 \cos \theta_{0} \cos \theta_{\mathrm{v}} \cos ^{4} \theta_{n}}$,

where

$P\left(\theta_{n}, W_{10 \mathrm{~m}}\right)=\frac{1}{\pi \sigma^{2}} \exp \left(-\frac{\tan ^{2} \theta_{n}}{\sigma^{2}}\right)$,

$\sigma^{2}=0.003+0.00512 W_{10 \mathrm{~m}}$,

$\theta_{n}=\arccos \left(\frac{\cos \theta_{\mathrm{v}}+\cos \theta_{0}}{2 \cos \omega}\right)$,

$\cos 2 \omega=\cos \theta_{\mathrm{v}} \cos \theta_{0}+\sin \theta_{\mathrm{v}} \sin \theta_{0} \cos \varphi$.

To describe the albedo of cloudy portions, we explored the application of two-stream equations as a function to ingest MODIS-retrieved cloud optical thickness $\tilde{\tau}$ and cloudtop effective radius $\overline{R_{\mathrm{e}}}$ through asymmetry parameter $g\left(\overline{R_{\mathrm{e}}}\right)$ or backscattering fraction $\beta\left(\overline{R_{\mathrm{e}}}\right)$, as explained in more detail in the following subsection. The following solutions are thoroughly described in Meador and Weaver (1980), which presents a unifying theoretical framework for a variety of two-stream cloud albedos based on coupled differential equations that describe upward- and downward-directed intensity fields. We considered two cloud albedos that proved useful for a range of cloud optical thicknesses (King and Harshvardhan, 1986): the Eddington approximation (Shettle and Weinman, 1970) and the Coakley-Chylek approximation (using solution I of Coakley and Chylek, 1975).

The Eddington approximation considered an incident flux explicitly in coupled equations and thus described diffuse intensity fields. Assuming conservative scattering (i.e., a single-scattering albedo of 1), a perfectly absorbing lower boundary $\left(\alpha^{\text {bottom }}=0\right)$, and no further influx at TOA, the analytical solution for cloud albedo was as follows, where $\mu_{0}=\cos \theta_{0}$ :

$\alpha^{\text {TwoStream }}=\frac{\frac{3}{4}(1-g) \tilde{\tau}-\frac{1}{4}\left(1-3 \mu_{0}\right)\left(1-e^{-\frac{\tilde{\tau}}{\mu_{0}}}\right)}{1+\frac{3}{4}(1-g) \tilde{\tau}}$.

The Coakley-Chylek approximation excluded the incident flux in differential equations, and thus its intensities referred to total radiation fields (i.e., direct and diffuse). Assuming conservative scattering, a perfectly absorbing lower boundary $\left(\alpha^{\text {bottom }}=0\right)$ and only a solar influx at TOA, the analytical solution for cloud albedo was

$\alpha^{\text {TwoStream }}=\frac{\frac{(1-g) \tilde{\tau}}{2}}{1+\frac{(1-g) \tilde{\tau}}{2}}$,

where $\beta$ was substituted with $\frac{(1-g)}{2}$ as done in textbook solutions (e.g., Bohren and Clothiaux, 2008). Using the CoakleyChylek approximation and a reflective lower boundary with albedo $\alpha^{\text {bottom }}>0$ (in this study $\alpha^{\text {bottom }}=\alpha^{\text {surface }}$ ), we produced following cloud albedo:

$\alpha^{\text {TwoStream }}=\frac{\alpha^{\text {ocean }}+\frac{\left(1-\alpha^{\text {ocean }}\right)(1-g) \tilde{\tau}}{2}}{1+\frac{\left(1-\alpha^{\text {ocean }}\right)(1-g) \tilde{\tau}}{2}}$.

Because it was unclear which solution could explain radiance fluctuations over narrow sun-observer geometries most successfully, we tested a variety of solutions in Sect. 4 .

Both ocean and cloud albedos (for up to two cloud layers) were used to calculate the footprint albedo, using clear fraction $f_{0}$ and cloud fractions of layer 1 and layer $2, f_{1}$ and $f_{2}$, respectively:

$$
\begin{aligned}
\alpha & =f_{0}\left(\alpha^{\text {ocean }}+r^{\text {SunGlint }}\right)+f_{1} \alpha_{1}^{\text {TwoStream }} \\
& +f_{2} \alpha_{2}^{\text {TwoStream }},
\end{aligned}
$$

where $f_{0}+f_{1}+f_{2}=1$.

\subsubsection{Statistical optimization}

Before comparing different two-stream approximations in Sect. 4, we performed two steps that ensured statistical optimization for each approximation. Finding an optimal $g\left(\overline{R_{\mathrm{e}}}\right)$ was shown to best capture radiance fluctuations per angular bin. Higher weights to a subset of data per angular bin - homogeneous clouds that were well retrieved - were used to facilitate consistency of radiances across bins. Both steps are explained in more detail below.

As shown in the previous subsection, we used two-stream cloud albedo to explain radiance fluctuations for narrow sunobserver geometries. Applied to all angular bins of an upward hemisphere, it was unclear which $g\left(\overline{R_{\mathrm{e}}}\right)$ to choose. Initial tests that used a $g\left(\overline{R_{\mathrm{e}}}\right)$ from Mie theory (see Fig. $2 \mathrm{~b}$ ) for all geometries proved suboptimal for some angular bins and left radiance residuals correlated to layer mean effective radius (not shown). We therefore decided to optimize $g\left(\overline{R_{\mathrm{e}}}\right)$ for each angular bin and for each cloud phase (liquid and ice). Inspired by the shape of Mie-calculated $g\left(\overline{R_{\mathrm{e}}}\right)$, we approximated $g\left(\overline{R_{\mathrm{e}}}\right)$ via a quadratic function,

$g\left(\overline{R_{\mathrm{e}}}\right)=a+b \overline{R_{\mathrm{e}}}+c{\overline{R_{\mathrm{e}}}}^{2}$,

and searched a three-dimensional grid, spanned by $a, b$, and $c$, for combinations that minimized the SD of radiance residuals. The search covered parameters $a, b$, and $c$ as listed in Table 2. As shown in Fig. 2a, we usually found a single optimum value that could minimize the SD of radiance residuals and that deviated from Mie calculations (Fig. 2b).

A second step aimed at using a subset of data that were consistent across angular bins. Looking into samples of individual angular bins, we observed stark variability in radiances that could be attributed to cloud horizontal heterogeneity (cloud homogeneity was approximated by $\nu=\frac{\bar{\tau}^{2}}{\sigma(\tau)^{2}}$; radiance residuals are shown in Fig. 2c). We suspected that 

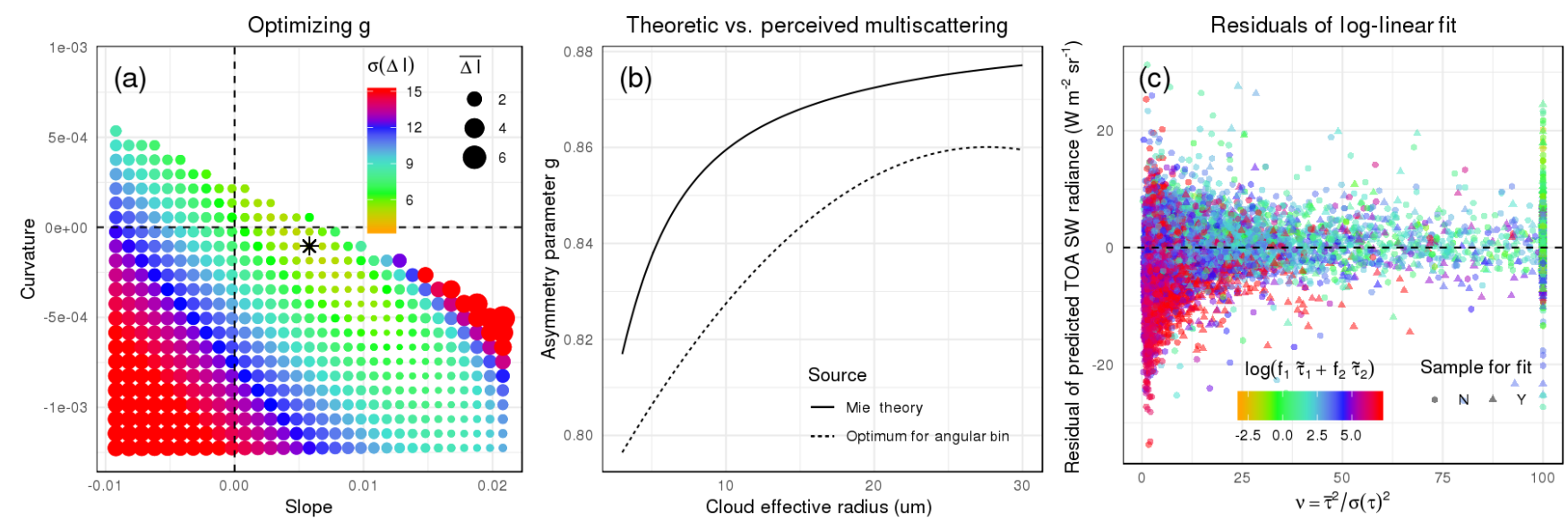

Figure 2. For the same angular bin as in Fig. 1, we present details of the proposed model that highlight essential steps aside from log-linear least-square fitting (Eq. 4). Panel (a) shows the search for an optimal $g\left(\overline{R_{\mathrm{e}}}\right)$ (as described in Sect. 3.2): we plotted a two-dimensional slice (showing $b$ and $c$ of Eq. 14) through the three-dimensional space (spanned by $a, b$, and $c$ ). Colors show the SD of radiance residuals and point size relates to model bias. The star marks the combination of $a, b$, and $c$ that produced the smallest residual SD and is considered optimal for this bin. Panel (b) compares the $g\left(\overline{R_{\mathrm{e}}}\right)$ of the determined optimal solution against Mie calculations. Panel (c) shows final radiance residuals against cloud homogeneity ( $x$ axis) and cloud optical depth (color). As described in Sect. 3.2.2., only homogeneous $(v>10)$ clouds which were well retrieved (MODIS-reported portion $\geq 80 \%)$ - marked as triangles in panel (c) - were conisidered for optimization of $g\left(\overline{R_{\mathrm{e}}}\right)$ and least-square fitting. Statistics and error metrics throughout the paper incorporate all samples.

Table 2. In search for optimal $g\left(\overline{R_{\mathrm{e}}}\right)$, we list the range (minimum and maximum) and step size for each parameter in Eq. (14).

\begin{tabular}{lrrr}
\hline Parameter & Minimum & Maximum & Step \\
\hline$a$ & -0.5 & 0.95 & 0.01 \\
$b$ & -0.01 & 0.01 & 0.0003 \\
$c$ & -0.00025 & 0.00025 & 0.000015 \\
\hline
\end{tabular}

clouds' three-dimensional structure caused tilted cloud facets that led to more or less reflective cloud portions (e.g., as accounted for in Scheck et al., 2018). In order to avoid an uncontrollable impact of cloud heterogeneity on final models, we decided to select homogeneous samples only for statistical optimization. As a threshold of homogeneity, we used $v>10$ (e.g., Barker et al., 1996; Kato et al., 2005). As shown in Table 3 per solar geometry, median homogeneity varied considerably across bins as well as cloud phase, and this resulted in ranging portions of data being selected. For optimization, we further limited selection to CERES footprints with quality flags indicating a confident retrieval of $80 \%$ or more of all cloudy MODIS pixels within a CERES footprint. This subset of samples served to optimize the above search for $g\left(\overline{R_{\mathrm{e}}}\right)$ and to find weights via least-square fitting (Eq. 4). To compute error metrics, we used all available samples. An example for the application of the log-linear model in shown in Fig. 1b.

\section{Results}

Radiance-predicting statistical models that capture narrow sun-observer geometries form the basis for empirical angular distribution models. And these statistical models fit observations from satellites, typically capturing how TOA SW radiances measured by a broadband radiometer change with scene type (defined by surface conditions as well as cloud and aerosol properties within the radiometer's footprint area) retrieved using a multispectral imager (see Sect. 2). To investigate whether a new approach, the proposed semiphysical log-linear model in Sect. 3.2, is a superior way to fit observations compared to the state-of-the-art approach, the sigmoidal fit described in Sect. 3.1, we took CERES Ed4SSF observations (Sect. 2) of liquid-phase clouds along the principal plane of an example solar geometry covering major scattering features of clouds and the ocean surface. We applied the sigmoidal fit as well as a variety of loglinear models, each using a different analytic solution of two-stream cloud albedo (Eqs. 10-12) that is used in this study as a framework to ingest MODIS-based cloud properties. Looking at the SD of radiance residuals per angular bin (in this study used as a measure of model uncertainty), the Coakley-Chylek approximation using a reflective lower boundary (Eq. 12) outperformed the sigmoidal fit for most bins by up to $1.5 \mathrm{~W} \mathrm{~m}^{-2} \mathrm{sr}^{-1}$ (shown in Fig. 3). Only the central portion of sun-glint-affected geometries remained best explained by sigmoidal fits (and accompanied lookup-table approach as laid out in Sect. 3.1). In contrast, the Coakley-Chylek approximation using a perfectly absorbing lower boundary (Eq. 11) or the Eddington approxima- 
Table 3. Per solar geometry $\theta_{0}$ and per cloud phase ( $\mathrm{L}$ - liquid, $\mathrm{I}$ - ice, $\mathrm{M}$ - mixed) as defined in Sect. 3.2, we show what portions of the upward hemisphere were covered with observations and how large the range of cloud homogeneity, above-cloud water vapor, and cloud-top effective radius was. The range lists minima and maxima of median values computed per angular bin within a hemisphere.

\begin{tabular}{|c|c|c|c|c|c|c|c|c|c|}
\hline \multirow[b]{2}{*}{$\theta_{0}^{\Delta}\left(\right.$ in $\left.^{\circ}\right)$} & \multicolumn{3}{|c|}{ Angular coverage } & \multicolumn{2}{|c|}{$v$} & \multicolumn{2}{|c|}{$\operatorname{ACWV}\left(\mathrm{kg} \mathrm{m}^{-2}\right)$} & \multicolumn{2}{|c|}{$\overline{R_{\mathrm{e}}}(\mu \mathrm{m})$} \\
\hline & $\mathrm{L}$ & I & M & $\mathrm{L}$ & I & $\mathrm{L}$ & I & $\mathrm{L}$ & I \\
\hline $6-8$ & 0.13 & 0.01 & 0.16 & $2.3-4.4$ & $5.2-12.2$ & $11.10-16.52$ & $0.03-0.04$ & $11.4-14.5$ & $39.8-45.7$ \\
\hline $8-10$ & 0.23 & 0.05 & 0.25 & $1.7-4.8$ & $4.3-10.4$ & $9.29-16.85$ & $0.02-0.05$ & $11.5-13.5$ & $38.6-47.8$ \\
\hline $10-12$ & 0.35 & 0.12 & 0.35 & $2.3-10.9$ & $3.7-15.8$ & $9.39-17.37$ & $0.02-0.06$ & $10.2-13.3$ & $38.5-49.6$ \\
\hline $12-14$ & 0.59 & 0.20 & 0.58 & $2.4-9.4$ & $3.0-17.1$ & $7.76-16.79$ & $0.02-0.06$ & $10.1-15.2$ & $38.7-47.5$ \\
\hline $14-16$ & 0.75 & 0.34 & 0.77 & $2.3-7.5$ & $3.2-22.4$ & $6.31-18.78$ & $0.02-0.08$ & $9.9-18.3$ & $36.7-74.3$ \\
\hline $16-18$ & 0.83 & 0.61 & 0.85 & $1.8-7.2$ & $3.4-24.9$ & $7.17-21.15$ & $0.02-0.06$ & $9.6-20.5$ & $36.3-47.3$ \\
\hline $18-20$ & 0.88 & 0.71 & 0.89 & $2.0-8.5$ & $3.1-20.2$ & 7.68-19.19 & $0.02-0.07$ & $9.5-20.0$ & $37.4-47.2$ \\
\hline $20-22$ & 0.91 & 0.76 & 0.93 & $2.0-9.1$ & $3.0-16.2$ & $6.77-19.97$ & $0.02-0.06$ & $10.0-19.5$ & $39.1-49.9$ \\
\hline $22-24$ & 0.93 & 0.79 & 0.95 & $2.0-9.1$ & $3.5-16.1$ & 7.00-18.79 & $0.02-0.06$ & $10.0-19.4$ & $38.6-49.0$ \\
\hline $24-26$ & 0.94 & 0.81 & 0.96 & $2.1-8.0$ & $3.6-15.5$ & $7.26-18.18$ & $0.02-0.07$ & $9.8-18.8$ & $40.1-61.0$ \\
\hline $26-28$ & 0.94 & 0.82 & 0.97 & $1.9-8.3$ & $3.2-15.5$ & $7.23-16.98$ & $0.02-0.06$ & $10.3-16.9$ & $40.2-51.9$ \\
\hline $28-30$ & 0.95 & 0.83 & 0.97 & $2.0-11.0$ & $3.3-15.8$ & $6.72-20.07$ & $0.02-0.07$ & $10.8-16.2$ & $39.5-51.6$ \\
\hline $30-32$ & 0.95 & 0.83 & 0.98 & $1.9-10.0$ & $3.4-16.4$ & $7.04-17.02$ & $0.02-0.08$ & $11.2-17.2$ & $40.7-73.2$ \\
\hline $32-34$ & 0.95 & 0.83 & 0.98 & $2.0-8.8$ & $3.1-17.0$ & 7.06-17.99 & $0.02-0.10$ & $11.6-16.9$ & $41.0-55.5$ \\
\hline $34-36$ & 0.96 & 0.83 & 0.98 & $2.0-8.8$ & $3.5-17.2$ & $6.44-18.76$ & $0.02-0.11$ & $11.8-16.0$ & $41.9-52.8$ \\
\hline $36-38$ & 0.96 & 0.83 & 0.98 & $1.9-6.9$ & $3.9-18.8$ & $6.66-18.18$ & $0.02-0.13$ & $11.9-15.8$ & $42.2-56.6$ \\
\hline $38-40$ & 0.95 & 0.83 & 0.98 & $2.0-7.7$ & $4.4-20.0$ & $6.61-16.88$ & $0.02-0.13$ & $11.7-15.8$ & $42.2-54.8$ \\
\hline $40-42$ & 0.95 & 0.83 & 0.98 & $1.9-7.7$ & $5.4-20.6$ & 5.55-19.06 & $0.03-0.14$ & $11.5-16.3$ & $43.6-55.8$ \\
\hline $42-44$ & 0.94 & 0.82 & 0.98 & $2.0-7.1$ & $5.0-20.3$ & $5.94-15.28$ & $0.03-0.16$ & $11.6-16.5$ & $43.0-62.8$ \\
\hline $44-46$ & 0.94 & 0.83 & 0.98 & $2.1-8.1$ & $5.7-20.5$ & $5.90-12.69$ & $0.04-0.15$ & $11.5-16.4$ & $43.7-74.4$ \\
\hline $46-48$ & 0.94 & 0.83 & 0.97 & $2.1-7.9$ & $6.0-19.4$ & $5.37-12.45$ & $0.03-0.15$ & $11.6-15.6$ & $44.3-78.2$ \\
\hline $48-50$ & 0.93 & 0.83 & 0.97 & $2.0-7.3$ & $6.1-17.9$ & $4.97-12.23$ & $0.04-0.16$ & $11.7-15.0$ & $45.3-76.1$ \\
\hline $50-52$ & 0.92 & 0.83 & 0.97 & $2.0-7.3$ & $5.7-18.1$ & $4.55-10.34$ & $0.05-0.16$ & $11.6-14.8$ & $44.6-80.0$ \\
\hline $52-54$ & 0.91 & 0.83 & 0.96 & $2.2-7.5$ & $5.6-16.6$ & 4.09-9.76 & $0.00-0.17$ & $11.8-15.0$ & $46.1-77.0$ \\
\hline $54-56$ & 0.90 & 0.83 & 0.96 & $2.1-7.0$ & $5.5-14.9$ & $3.88-8.67$ & $0.04-0.16$ & $11.0-15.7$ & $46.1-78.6$ \\
\hline $56-58$ & 0.89 & 0.83 & 0.96 & $3.0-7.4$ & $5.2-13.3$ & $3.30-8.88$ & $0.05-0.15$ & $10.8-15.0$ & $45.0-76.2$ \\
\hline $58-60$ & 0.88 & 0.83 & 0.96 & $3.1-8.8$ & $4.6-12.4$ & $2.99-8.77$ & $0.05-0.15$ & $10.7-15.1$ & $44.0-76.9$ \\
\hline $60-62$ & 0.87 & 0.84 & 0.95 & $2.8-8.7$ & $4.8-11.7$ & $3.24-10.05$ & $0.05-0.14$ & $11.2-15.2$ & $43.8-76.8$ \\
\hline $62-64$ & 0.86 & 0.83 & 0.95 & $3.4-8.7$ & $4.5-13.3$ & $2.74-7.62$ & $0.04-0.15$ & $11.5-14.9$ & $44.1-76.2$ \\
\hline $64-66$ & 0.85 & 0.84 & 0.94 & $3.5-9.6$ & $3.9-11.7$ & $2.32-7.73$ & $0.04-0.15$ & $11.5-14.7$ & $42.9-79.5$ \\
\hline $66-68$ & 0.85 & 0.84 & 0.94 & $3.5-9.3$ & $3.5-10.6$ & $2.37-8.56$ & $0.05-0.15$ & $11.6-15.2$ & $44.1-82.2$ \\
\hline $68-70$ & 0.84 & 0.84 & 0.93 & $3.8-11.2$ & $3.2-10.1$ & $2.30-9.00$ & $0.05-0.15$ & $11.5-15.3$ & $41.0-75.6$ \\
\hline 70-72 & 0.83 & 0.83 & 0.93 & $3.9-11.6$ & $2.7-10.2$ & $2.45-7.02$ & $0.05-0.16$ & $11.7-15.1$ & $42.3-76.1$ \\
\hline $72-74$ & 0.82 & 0.82 & 0.92 & $3.8-12.7$ & $2.2-9.1$ & $2.05-6.44$ & $0.06-0.15$ & $11.8-15.8$ & $40.6-86.2$ \\
\hline $74-76$ & 0.80 & 0.81 & 0.91 & $4.0-10.9$ & $1.8-10.0$ & $1.98-7.46$ & $0.04-0.15$ & $11.1-16.1$ & $40.0-76.6$ \\
\hline $76-78$ & 0.79 & 0.79 & 0.90 & $3.8-13.0$ & $1.5-8.8$ & $1.87-9.36$ & $0.06-0.15$ & $11.6-15.8$ & $39.2-88.6$ \\
\hline $78-80$ & 0.78 & 0.77 & 0.90 & $3.5-13.2$ & $1.1-8.0$ & $1.50-9.97$ & $0.04-0.14$ & $11.5-15.9$ & $35.9-88.6$ \\
\hline $80-82$ & 0.75 & 0.75 & 0.89 & $3.4-11.7$ & $1.2-8.2$ & $1.60-8.82$ & $0.04-0.13$ & $11.5-15.1$ & $29.5-88.5$ \\
\hline
\end{tabular}

tion (Eq. 10) performed only equally well or worse than sigmoidal fits.

To ensure that the Coakley-Chylek approximation using a reflective lower boundary performed well for other sunobserver geometries, we processed all angular bins that contained more than 100 samples. As Table 3 shows, this covered between 13 and $96 \%$ of all angular bins. We found that for liquid clouds (top panels of Fig. 4) and $\theta_{0} \sim 20-70^{\circ}$, more than half of the bins were better explained by the loglinear approach and errors were reduced by up to $13.2 \%$. For solar geometries $\theta_{0}>40^{\circ}$, bins in sun-glint-affected ge- ometries (constituting a portion of all bins in a hemisphere between $10 \%$ for a $\theta_{0} \sim 20^{\circ}$ and $1 \%$ for a $\theta_{0} \sim 75^{\circ}$ ) caused higher uncertainties in log-linear models, increasing with solar zenith angle and higher by up to $60 \%$ compared to the sigmoidal approach. For solar geometries $\theta_{0}<20^{\circ}$, on the other hand, we found bins outside the sun glint - i.e., mostly slant observation angles - were best treated with the sigmoidal approach. A few footprints (indicated by circle size) of the top row were treated as mixed in the log-linear model and will be evaluated further below. With these limitations in mind, we use the Coakley-Chylek approximation using a reflective 


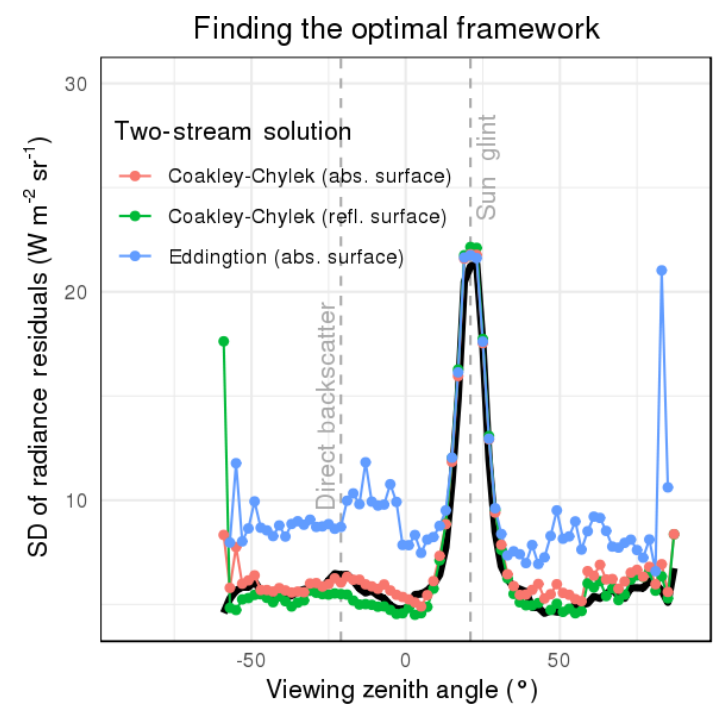

Figure 3. Applied to angular bins of the principal plane for $\theta_{0} \in$ $\left[20^{\circ}, 22^{\circ}\right]$, we test a variety of two-stream solutions for cloud albedo (Eqs. 10-12) as input to log-linear models as presented in Eq. (4). This plot shows the SD of resulting residuals and compares against the state-of-the-art sigmoidal fit (black line). As labeled, gray dashed lines mark the position of sun-glint and direct backscatter.

lower boundary standard as two-stream cloud albedo for the remainder of this study.

To determine whether the log-linear approach predicted plausible radiance fields, we tested it on a variety of scenarios. When applied to a range of cloud optical thicknesses, we found a similar radiance response compared to the sigmoidal fit (Fig. 5b). Setting cloud fraction to zero $\left(f_{1}=f_{2}=0\right)$ and using a range of $10 \mathrm{~m}$ wind speeds, log-linear and sigmoidal models produced again comparable radiance fields (Fig. 5c). This shows that the sensitivities of the state-of-the-art approach were captured by log-linear models. When varying cloud-top effective radius - a newly added sensitivity - we found radiances grew as droplet size increased (leaving cloud optical thickness constant; shown in Fig. 5e). With a focus on single-scattering features, we found the cloud glory (centered around the direct backscatter) to widen and the cloud glory (positioned about $20^{\circ}$ away from the backscatter) to shift towards the direct backscatter as effective radii became smaller. This observation is corroborated by Mie calculations of scattering phase functions (e.g., Fig. 1 in Tornow et al., 2018). The newly introduced concept of bin-wise optimized asymmetry parameters (Sect. 3.2.2) made changing cloud bow and glory possible, and $g\left(\overline{R_{\mathrm{e}}}\right)$ exhibited a symmetry left and right of the direct backscatter between $\theta_{\mathrm{v}}$ of -50 and $0^{\circ}$ (Fig. 5f). For a range of above-cloud water vapor (Fig. 5d) another newly added sensitivity - we observed that smaller loads produced higher radiances and found a slight increase in sensitivity with larger $\theta_{\mathrm{v}}$.
We also tested log-linear models on observations of icephase clouds. We found that model uncertainties outside the sun glint were of similar magnitude to sigmoidal fits (Fig. 4, bottom panels). Possible reasons will be discussed in Sect. 5 . Similar to liquid-phase clouds, angular geometries affected by sun glint showed worse performance when using loglinear models, increasing residuals by up to $30 \%$. Like the liquid phase, predicted radiances increased with smaller ice crystal radii. However, distinct scattering features were absent (not shown) - possibly a result of ice clouds' rich variety of crystal shapes (e.g., Zhang et al., 1999; Baum et al., 2005), which was unaccounted for. The response to abovecloud water vapor was consistent and covered much of the lower levels (0.03-0.17 $\mathrm{kg} \mathrm{m}^{-2}$; see Table 3).

Roughly $50 \%$ of all CERES footprints cover both a liquid and an ice cloud and have been treated separately as mixed phase. The proposed log-linear approach allows us to handle mixed-phase cases fundamentally differently. Instead of a footprint-effective optical depth (as used in Eq. 2), we can produce a footprint-effective albedo (Eq. 13) and account not only for cloud macrophysical $\left(f_{1 / 2}, \tilde{\tau}_{1 / 2}\right)$ but also for microphysical $\left(\overline{R_{\mathrm{e}} 1 / 2}\right)$ changes. Optimized asymmetry parameters from pure liquid and pure ice cases (Fig. 6b) were reused to describe the cloud albedo of respective cloud phase within each mixed-phase CERES footprint. Hence only $A, B$, and $C$ from Eq. (4) needed to be estimated. Figure 6a illustrates the reduction in model uncertainty for many bins and of up to $2.5 \mathrm{~W} \mathrm{~m}^{-2} \mathrm{sr}^{-1}$ when using the log-linear approach. Once again, the center of sun glint remained best captured by the sigmoidal approach, especially for solar zenith angles beyond $50^{\circ}$ where semiphysical models produce up to $55 \%$ higher residuals. Using a cloud-phase-specific albedo allowed us to account for radiance changes with varying amount of liquid vs. ice fraction within a footprint. Figure $6 c$ shows radiance predictions for different liquid-ice proportions (which could not be captured by the state-of-the-art approach) and that both approaches agree for $50 \%$ liquid and $50 \%$ ice cloud footprints for the backscattering direction and $75 \%$ liquid and $25 \%$ ice cloud fractions for much of the forward scattering direction. indicating that sampled footprints shifted in liquid-to-ice proportions along the principal plane. Figure $6 \mathrm{~d}$ shows the sigmoidal fit's sensitivity to ranging cloud optical depth was captured by the log-linear approach. Looking at all available sun-observer geometries (Fig. 4, middle panels) for solar geometries between $\theta_{0} \sim 20$ and $70^{\circ}$, we found model uncertainty of most bins reduced by as much as $35.8 \%$.

Across all solar and viewing geometries, we calculated the median change in uncertainty when using the log-linear approach over the state-of-the-art approach to be $-5.76 \%$ for liquid-phase clouds, $+0.34 \%$ for ice-phase clouds, and $-10.81 \%$ when both phases were present.

In summary, we showed that the proposed log-linear model had the ability to outperform the existing sigmoidal approach in capturing CERES radiance fluctuations per an- 


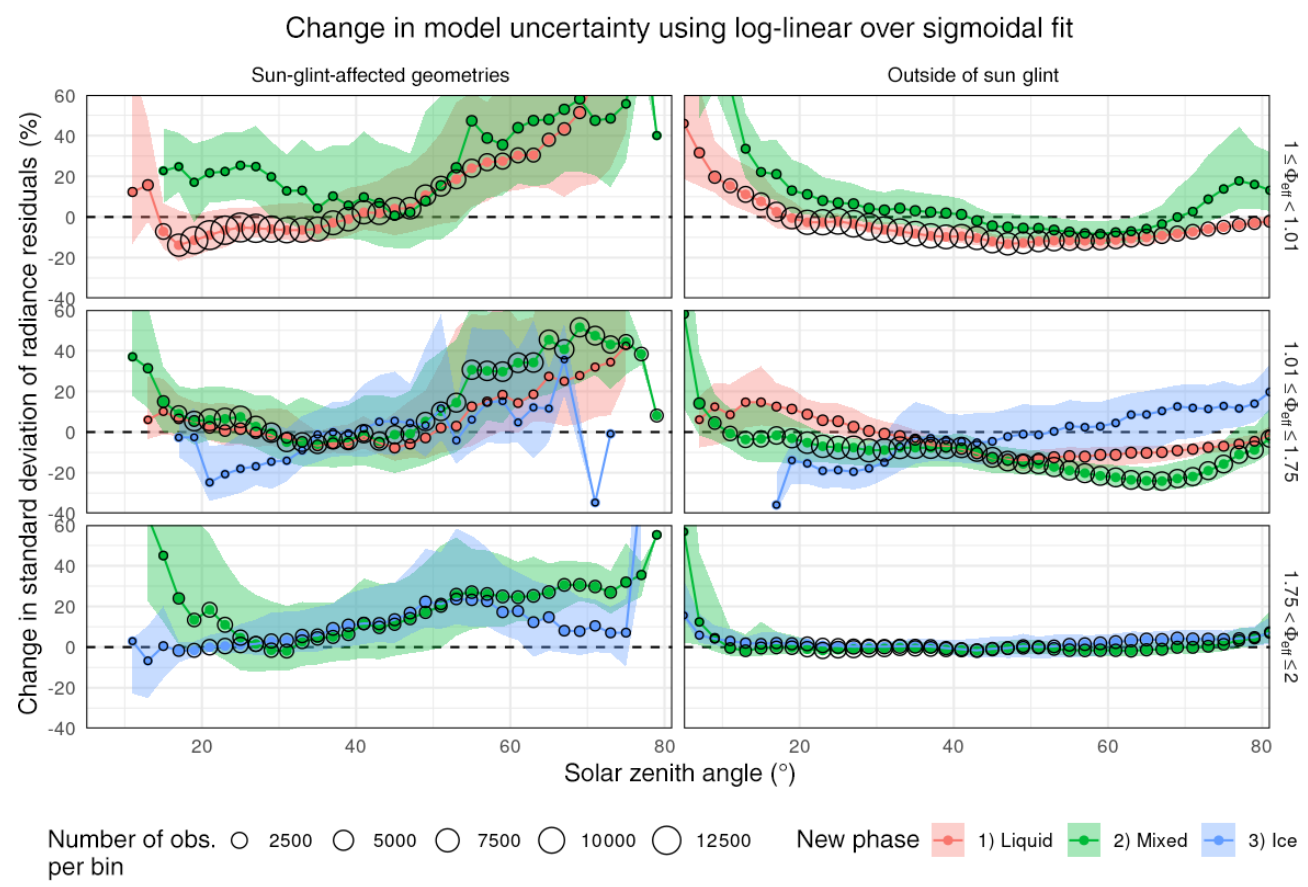

Figure 4. Using all observed angular bins within $\theta_{0} \in\left[6^{\circ}, 82^{\circ}\right]$, we show how radiance residuals from proposed log-linear models compare against state-of-the-art sigmoidal fits. Results are presented by the CERES-defined cloud phase (vertically), by the newly defined phase (colors), and by whether the angular geometry is affected by sun glint (left) or free of sun glint (right). We show relative change in model uncertainty: $\delta=\left[\sigma\left(\Delta I^{\text {LogLinear }}\right)-\sigma\left(\Delta I^{\text {Sigmoidal }}\right)\right] / \sigma\left(\Delta I^{\text {Sigmoidal }}\right) \cdot 100 \%$. Consequently, negative values relate to a better performance of the log-linear model, while positive values mark a better performance by the state-of-the-art methodology. Solid lines and dots mark the 50th percentile and shades show the interquartile range between the 25 th and 75 th percentiles. Point size relates to the average number of observations per angular bin. The dashed black line marks zero change.

gular bin. For most geometries it produced lower uncertainties, added new radiance sensitivities, and allowed us to treat mixed-phase footprints in a fundamentally different manner. Drawbacks were typically found for geometries affected by sun glint.

\section{Conclusions}

Statistical models that capture measurements of TOA SW radiances as a function of corresponding scene type for narrow sun-observer geometries are the basis for angular distribution models. In this study, we introduced a new alternative that incorporated additional parameters - namely cloud-top effective radius and above-cloud water vapor - via a semiphysical log-linear approach. We found this new approach to better explain radiance fluctuations for the majority of observed geometries and to produce plausible radiance fields. Weaker performance than the state-of-the-art approach was generally observed for solar zenith angles lower than $20^{\circ}$ and for sun-glint-affected geometries that constitute between $1 \%$ and $10 \%$ of the hemispheric radiance field.

Incorporating additional parameters that help explain radiance fluctuations may have minimized sampling bias. Ranges in effective radius or above-cloud water vapor varied across bins, and ignoring this variation can cause a radiance bias in individual angular bins. Even accounting for parameters that may not affect TOA anisotropy, such as cloud horizontal heterogeneity, has the potential to minimize sampling biases. We found varying portions of heterogeneous samples across bins and suspect that their variation in radiance (cf. Fig. 2c) failed to cancel out. Thus, giving higher (or all) weight to homogeneous samples during regression, as done in this study, should eliminate any sampling bias.

The inclusion of cloud-top effective radius and abovecloud water vapor was successful, as evidenced by reduced radiance residuals and credible radiance fields. We failed to reduce radiance residuals for ice-phase clouds and made the following observations looking at ice cloud samples. First, among collected observations, we found footprints to mostly contain homogeneous ice clouds. Second, ice clouds had only small loads of water vapor aloft. Lastly, there was an absence of distinct single-scattering features. We suspect that these are characteristics that drive the potential reduction of radiance residuals and that liquid clouds samples, having near-asymmetric properties (few homogeneous samples, large loads of water vapor aloft, distinct scattering features), benefitted especially from this new approach. 

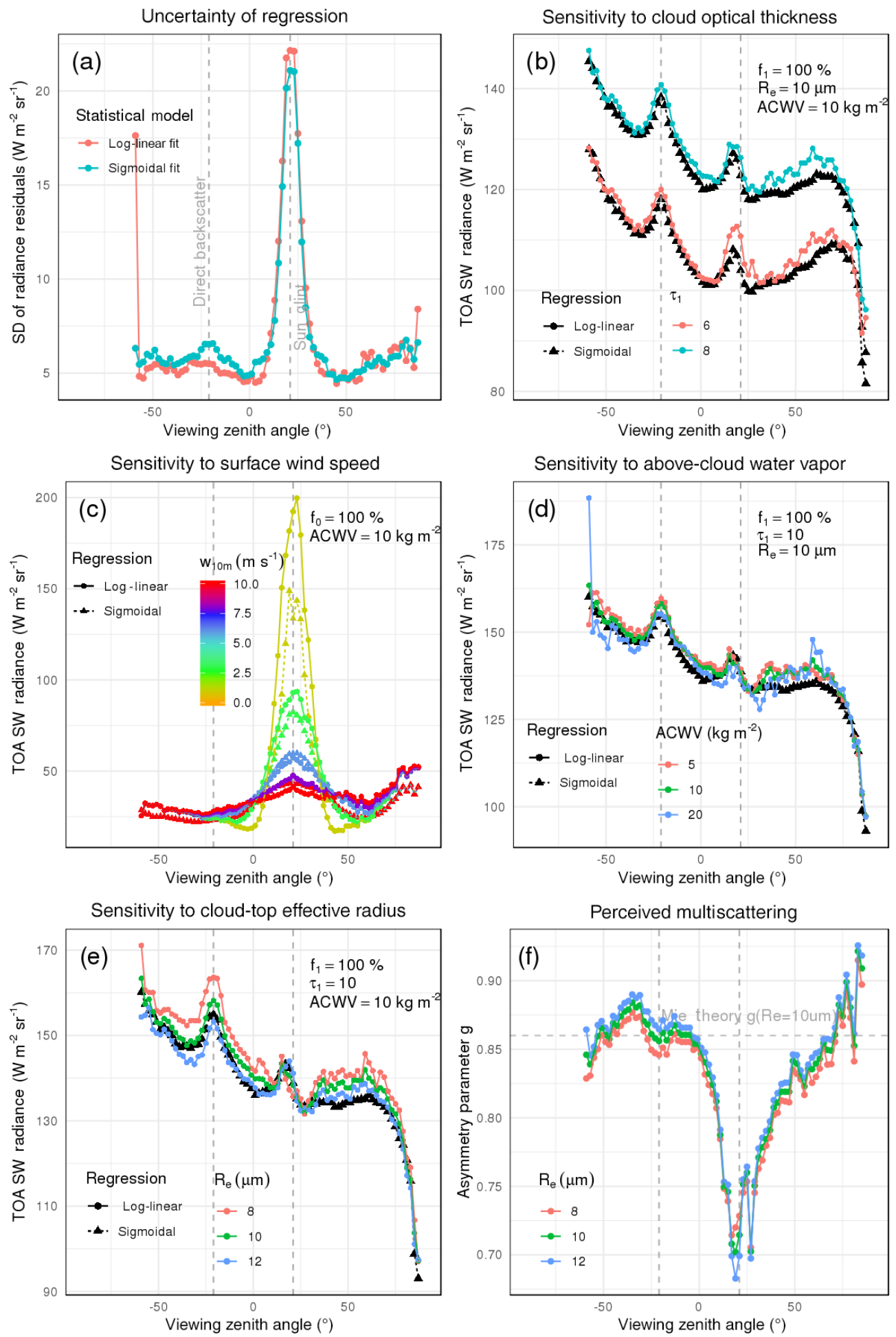

Figure 5. For angular bins along the principal plane for $\theta_{0} \in\left[20^{\circ}, 22^{\circ}\right]$ containing liquid-phase footprints, we present error metrics and sensitivities of proposed log-linear vs. state-of-the-art sigmoidal fits. Panel (a) shows the SD of residuals; colors mark the type of fit. Panel (b) displays the optimal $g\left(\overline{R_{\mathrm{e}}}\right)$ for three $R_{\mathrm{e}}$ (by color). Panels (c), (d), and (e) demonstrate predicted radiances by both fits for varying cloud optical thickness (c), cloud-top effective radius (d), and above-cloud water vapor (e). Predictions from log-linear fits are colored, while predictions from sigmoidal fits are shown in black. Panel (f) presents the response of both fits to a variety of surface wind speeds. Properties held constant in panels (c), (d), (e), and (f) are listed in the each panel's top-right corner. 

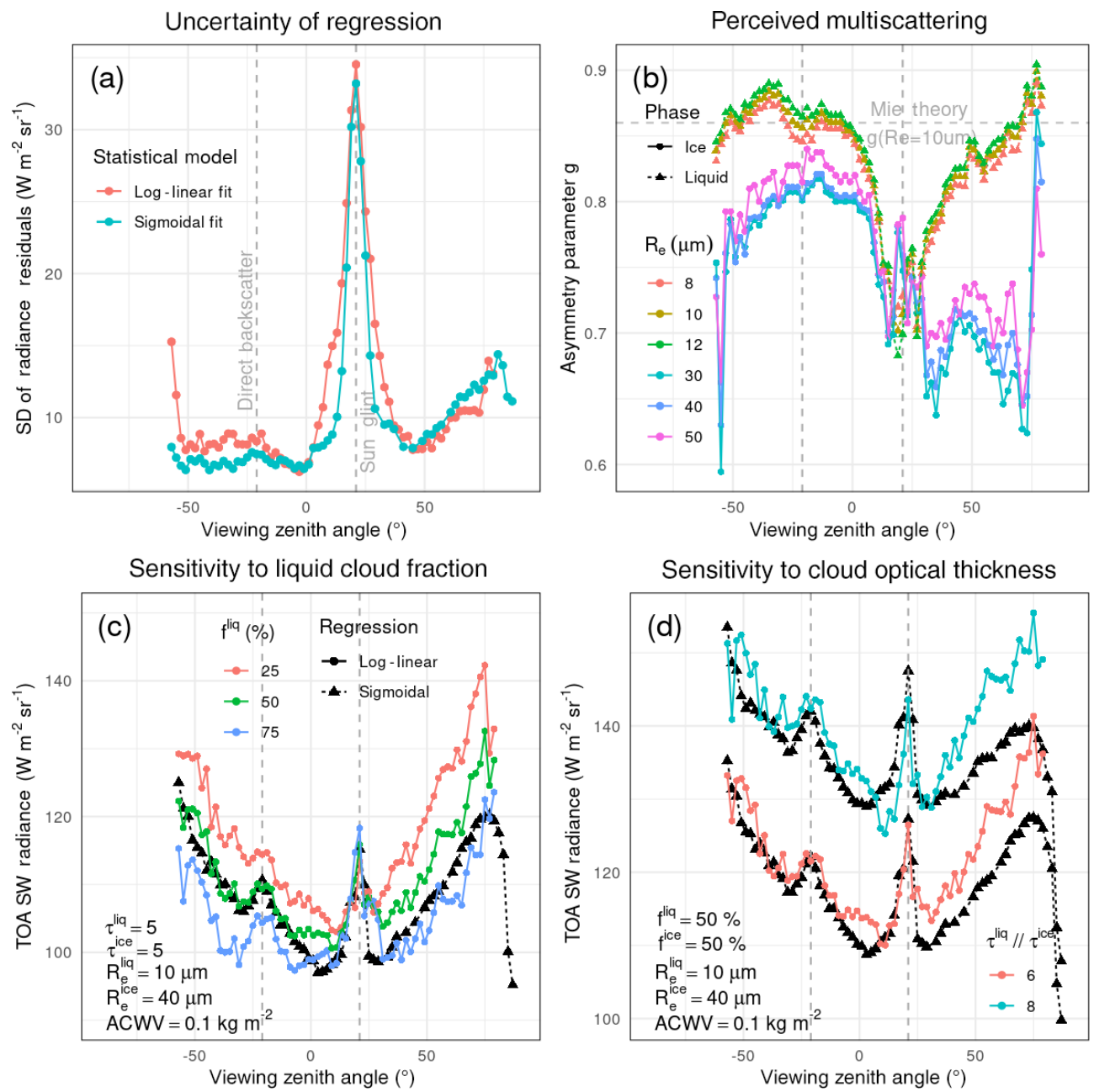

Figure 6. For angular bins along the principal plane for $\theta_{0} \in\left[20^{\circ}, 22^{\circ}\right]$, we show details for mixed-phase footprints. Panel (a) presents the $\mathrm{SD}$ of residuals (colors mark the type of fit). Panel (b) shows optimal $g\left(\overline{R_{\mathrm{e}}}\right)$ from pure ice- and liquid-phase footprints employed for mixedphase cases (colors mark liquid and ice particle effective radius). Panels (c) and (d) show predicted radiances for liquid cloud fraction (c) and cloud optical thickness (d). In panels (c) and (d), we show log-linear fits in color and sigmoidal fits in black. Quantities left constant are shown in the bottom-left corner.

We successfully used a theoretic framework - inspired by radiative transfer approximations designed for hemispheric averages - and applied it to narrow sun-observer geometries. A derived byproduct, the asymmetry parameter $g\left(\overline{R_{\mathrm{e}}} \mid \theta_{0}^{\Delta}, \theta_{\mathrm{v}}^{\Delta}, \varphi^{\Delta}\right)$, captured observer-specific multiscattering. Could this byproduct contain information that allows inference on multiscattering properties? Monte Carlo radiative transfer simulations may help in answering this. Future work should simulate radiances, derive simulation-based $g\left(\overline{R_{\mathrm{e}}} \mid \theta_{0}^{\Delta}, \theta_{\mathrm{v}}^{\Delta}, \varphi^{\Delta}\right)$, and extract additional properties, such as photon path length or number of scattering events.

Statistical models allow finding scene properties that produce similar radiative responses (often referred to as similarity conditions). Like the state-of-art approach, where different combinations of cloud fraction and cloud optical thickness produced similar radiances, the new semiphysical ap- proach added cloud particle size and above-cloud absorber mass to parameter combinations. A similarity condition explaining albedo through adjusted optical thickness, $(1-g) \tilde{\tau}$, was found earlier using simulations (e.g., van de Hulst, 1996). To our knowledge, this is the first time adjusted optical thickness (here employed in the framework of two-stream albedo) has been used to capture similarities of observed radiances.

The proposed semiphysical approach can easily be applied to land surfaces. Imager-based bidirectional reflectance distribution function (BRDF) products, such as MCD43GF (MODIS BRDF and Albedo Global Gap-Filled, Snow-Free Version 6; https://doi.org/10.5067/MODIS/MCD43GF.006, Schaaf, 2019), could provide land surface albedo and surface bidirectional reflectance in order to determine each observation's footprint albedo. Future efforts should test if this ap- 
plication over land can compete with CERES' separate treatment by latitude-longitude boxes. Recent efforts that demonstrated circumvention of this regional separation for clearsky ADMs by using MCD43GF instead indicated a positive outcome (Tornow et al., 2019).

Lastly, we hope this new log-linear approach will form the basis of future angular distribution models. In particular, we expect that cloudy scenes of microphysical extremes (i.e., clouds consisting of very small or very large droplets) observed from the backscattering direction will benefit from radiance-to-flux conversion using new models. More accurate estimates of instantaneous fluxes should benefit EarthCARE' studies of cloud-radiative processes regarding both water and energy fluxes. We are currently examining this impact on instantaneous fluxes as well as the propagation of updated flux estimates into daily and monthly flux products.

Data availability. CERES Ed4SSF data were downloaded through https://ceres.larc.nasa.gov/data/ (NASA Langley Research Center, 2020) and ERA20C was accessed via https://apps.ecmwf.int/ datasets/data/era20c-daily/ (ECMWF, 2020).

Author contributions. FT had the idea, designed the experiment, and conducted the research. CD, HWB, and RP had a major influence on the development of the methodology through discussion. CD and HWB further helped revise this paper. JF provided essential resources for data processing and evaluation.

Competing interests. The authors declare that they have no conflict of interest.

Acknowledgements. We thank Jason N. S. Cole, Almudena Velazquez Blazquez, Tobias Wehr, and all other members of the CLARA team as well as colleagues at the Institute for Space Sciences at Freie Universität Berlin for the helpful discussion. We further thank the Atmospheric Sciences Data Center at the National Aeronautics and Space Administration Langley Research Center for providing the Clouds and the Earth's Radiant Energy System Single Scanner Footprint TOA/Surface Fluxes and Clouds data product. We specifically thank Wenying Su for providing us with details on the CERES methodology. We further thank two anonymous referees very much for their feedback that helped to improve this paper substantially.

Financial support. This research has been supported by the European Space Agency (grant no. 4000112019/14/NL/CT).

We acknowledge support from the Open Access Publication Initiative of Freie Universität Berlin.
Review statement. This paper was edited by Alexander Kokhanovsky and reviewed by two anonymous referees.

\section{References}

Barker, H. W. and Wehr, T.: Computation of Solar Radiative Fluxes by 1D and 3D Methods Using Cloudy Atmospheres Inferred from A-train Satellite Data, Surv. Geophys., 33, 657-676, https://doi.org/10.1007/s10712-011-9164-9, 2012.

Barker, H. W., Wielicki, B. A., and Parker, L.: A Parameterization for Computing Grid-Averaged Solar Fluxes for Inhomogeneous Marine Boundary Layer Clouds. Part II: Validation Using Satellite Data, J. Atmos. Sci., 53, 2304-2316, https://doi.org/10.1175/15200469(1996)053<2304:APFCGA>2.0.CO;2, 1996.

Barker, H. W., Jerg, M. P., Wehr, T., Kato, S., Donovan, D. P., and Hogan, R. J.: A 3D cloud-construction algorithm for the EarthCARE satellite mission, Q. J. Roy. Meteor. Soc., 137, 10421058, https://doi.org/10.1002/qj.824, 2011.

Baum, B. A., Heymsfield, A. J., Yang, P., and Bedka, S. T.: Bulk Scattering Properties for the Remote Sensing of Ice Clouds. Part I: Microphysical Data and Models, J. Appl. Meteorol., 44, 18851895, https://doi.org/10.1175/JAM2308.1, 2005.

Bender, F. A.-M., Rohde, H., Charloson, R. J., Ekman, A. M. L., and Loeb, N.: 22 views of the global albedo - comparison between $20 \mathrm{GCMs}$ and two satellites, Tellus A, 58, 320-330, https://doi.org/10.1111/j.1600-0870.2006.00181.x, 2006.

Bohren, C. F. and Clothiaux, E. E.: Scattering: The Life of Photons, Chap. 3, John Wiley and Sons, Ltd, 125-184, https://doi.org/10.1002/9783527618620.ch3, 2008.

Bolton, D.: The Computation of Equivalent Potential Temperature, Mon. Weather Rev., 108, 1046-1053, https://doi.org/10.1175/15200493(1980)108<1046:TCOEPT>2.0.CO;2, 1980.

Boucher, O., Randall, D., Artaxo, P., Bretherton, C., Feingold, G., Forster, P., Kerminen, V.-M., Kondo, Y., Liao, H., Lohmann, U., Rasch, P., Satheesh, S., Sherwood, S., Stevens, B., and Zhang, X.: Clouds and Aerosols, Sect. 7, Cambridge University Press, Cambridge, United Kingdom and New York, NY, USA, https://doi.org/10.1017/CBO9781107415324.016, 571658, 2013.

Calisto, M., Folini, D., Wild, M., and Bengtsson, L.: Cloud radiative forcing intercomparison between fully coupled CMIP5 models and CERES satellite data, Ann. Geophys., 32, 793-807, https://doi.org/10.5194/angeo-32-793-2014, 2014.

Coakley, J. A. and Chylek, P.: The Two-Stream Approximation in Radiative Transfer: Including the Angle of the Incident Radiation, J. Atmos. Sci., 32, 409-418, https://doi.org/10.1175/15200469(1975)032<0409:TTSAIR>2.0.CO;2, 1975.

Cox, C. and Munk, W.: Measurement of the Roughness of the Sea Surface from Photographs of the Sun's Glitter, J. Opt. Soc. Am., 44, 838-850, https://doi.org/10.1364/JOSA.44.000838, 1954.

European Centre for Medium-Range Weather Forecasts (ECMWF): ERA-20C Daily Dataset, available at: https://apps.ecmwf.int/ datasets/data/era20c-daily, last access: 17 July 2020.

Gao, M., Huang, X., Yang, P., and Kattawar, G. W.: Angular distribution of diffuse reflectance from incoherent multi- 
ple scattering in tur bid media, Appl. Optics, 52, 5869-5879, https://doi.org/10.1364/AO.52.005869, 2013.

Illingworth, A. J., Barker, H. W., Beljaars, A., Chepfer, H., Delanoe, J., Domenech, C., Donovan, D. P., Fukuda, S., Hirakata, M., Hogan, R. J., Huenerbein, A., Kollias, P., Kubota, T., Nakajima, T., Nakajima, T. Y., Nishizawa, T., Ohno, Y., Okamoto, H., Oki, R., Sato, K., Satoh, M., Wandinger, U., Wehr, T., and van Zadelhoff, G.: The EarthCARE Satellite: the next step forward in global measurements of clouds, aerosols, precipitation and radiation, B. Am. Meteorol. Soc, 96, 1311-1332, https://doi.org/10.1175/BAMS-D-12-00227.1, 2015.

Kato, S., Rose, F. G., and Charlock, T. P.: Computation of Domain-Averaged Irradiance Using Satellite-Derived Cloud Properties, J. Atmos. Ocean. Tech., 22, 146-164, https://doi.org/10.1175/JTECH-1694.1, 2005.

King, M. D. and Harshvardhan: Comparative Accuracy of Selected Multiple Scattering Approximations, J. Atmos. Sci., 43, 784-801, https://doi.org/10.1175/15200469(1986)043<0784:CAOSMS>2.0.CO;2, 1986.

Li, J., Yi, Y., Minnis, P., Huang, J., Yan, H., Ma, Y., Wang, W., and Ayers, J. K.: Radiative effect differences between multilayered and single-layer clouds derived from CERES, CALIPSO, and CloudSat data, J. Quant. Spectrosc. Ra., 112, 361-375, https://doi.org/10.1016/j.jqsrt.2010.10.006, international Symposium on Atmospheric Light Scattering and Remote Sensing (ISALSaRS'09), 2011.

Loeb, N. G. and Manalo-Smith, N.: Top-of-Atmosphere Direct Radiative Effect of Aerosols over Global Oceans from Merged CERES and MODIS Observations, J. Climate, 18, 3506-3526, https://doi.org/10.1175/JCLI3504.1, 2005.

Loeb, N. G., Manalo-Smith, N., Kato, S., Miller, W. F., Gupta, S. K., Minnis, P., and Wielicki, B. A.: Angular Distribution Models for Top-of-Atmosphere Radiative Flux Estimation from the Clouds and the Earth's Radiant Energy System Instrument on the Tropical Rainfall Measuring Mission Satellite. Part I: Methodology, J. Appl. Meteorol., 42, 240-265, https://doi.org/10.1175/15200450(2003)042<0240:ADMFTO>2.0.CO;2, 2003.

Loeb, N. G., Kato, S., Loukachine, K., and Manalo-Smith, N.: Angular Distribution Models for Top-of-Atmosphere Radiative Flux Estimation from the Clouds and the Earth's Radiant Energy System Instrument on the Terra Satellite. Part I: Methodology, J. Atmos. Ocean. Tech., 22, 338-351, https://doi.org/10.1175/JTECH1712.1, 2005.

Loeb, N. G., Doelling, D. R., Wang, H., Su, W., Nguyen, C., Corbett, J. G., Liang, L., Mitrescu, C., Rose, F. G., and Kato, S.: Clouds and the Earth's Radiant Energy System (CERES) Energy Balanced and Filled (EBAF) Top-ofAtmosphere (TOA) Edition-4.0 Data Product, J. Climate, 31, 895-918, https://doi.org/10.1175/JCLI-D-17-0208.1, 2018.

Meador, W. E. and Weaver, W. R.: Two-Stream Approximations to Radiative Transfer in Planetary Atmospheres: A Unified Description of Existing Methods and a New Improvement, J. Atmos. Sci., 37, 630-643, https://doi.org/10.1175/15200469(1980)037<0630:TSATRT>2.0.CO;2, 1980.

Nam, C., Bony, S., Dufresne, J.-L., and Chepfer, H.: The "too few, too bright" tropical low-cloud problem in CMIP5 models, Geophys. Res. Lett., 39, L21801, https://doi.org/10.1029/2012GL053421, 2012.
NASA Langley Research Center: CERES Data Products, available at: https://ceres.larc.nasa.gov/data/, last access: 17 July 2020.

Poli, P., Hersbach, H., Dee, D. P., Berrisford, P., Simmons, A. J., Vitart, F., Laloyaux, P., Tan, D. G. H., Peubey, C., Thépaut, J.-N., Trémolet, Y., Hólm, E. V., Bonavita, M., Isaksen, L., and Fisher, M.: ERA-20C: An Atmospheric Reanalysis of the Twentieth Century, J. Climate, 29, 4083-4097, https://doi.org/10.1175/JCLI-D-15-0556.1, 2016.

Schaaf, C.: MODIS/Terra+Aqua BRDF/Albedo Gap-Filled Snow-Free Daily L3 Global 30ArcSec CMG V006, distributed by NASA EOSDIS Land Processes DAAC, https://doi.org/10.5067/MODIS/MCD43GF.006, 2019.

Scheck, L., Weissmann, M., and Mayer, B.: Efficient Methods to Account for Cloud-Top Inclination and Cloud Overlap in Synthetic Visible Satellite Images, J. Atmos. Ocean. Tech., 35, 665685, https://doi.org/10.1175/JTECH-D-17-0057.1, 2018.

Shettle, E. P. and Weinman, J. A.: The Transfer of Solar Irradiance Through Inhomogeneous Turbid Atmospheres Evaluated by Eddington's Approximation, J. Atmos. Sci., 27, 1048-1055, https://doi.org/10.1175/15200469(1970)027<1048:TTOSIT>2.0.CO;2, 1970.

Smith, G. L., Green, R. N., Raschke, E., Avis, L. M., Suttles, J. T., Wielicki, B. A., and Davies, R.: Inversion methods for satellite studies of the Earth's Radiation Budget: Development of algorithms for the ERBE Mission, Rev. Geophys., 24, 407-421, https://doi.org/10.1029/RG024i002p00407, 1986.

Stephens, G., Li, J., and Wild, M.: An update on Earth's energy balance in light of the latest global observations, Nat. Geosci., 5, 691-696, https://doi.org/10.1038/ngeo1580, 2012.

Su, W., Corbett, J., Eitzen, Z., and Liang, L.: Next-generation angular distribution models for top-of-atmosphere radiative flux calculation from CERES instruments: methodology, Atmos. Meas. Tech., 8, 611-632, https://doi.org/10.5194/amt-8-6112015, 2015.

Sun-Mack, S., Minnis, P., Chen, Y., Doelling, D. R., Scarino, B. R., Haney, C. O., and Smith, W. L. J.: Calibration Changes to Terra MODIS Collection-5 Radiances for CERES Edition 4 Cloud Retrievals, IEEE T. Geosci. Remote, 56, 6016-6032, https://doi.org/10.1109/TGRS.2018.2829902, 2018.

Suttles, J. T., Green, R. N., Minnis, P., Smith, G., Staylor, W. F., Wielicki, B., J., W. I., Young, D., Taylor, V. R., and Stowe, L. L.: Angular radiation models for Earth-atmosphere systems, Volume 1 - Shortwave Radiation, Tech. Rep. 1184, NASA, Washington, D.C., USA, 1988.

Thorsen, T. J., Kato, S., Loeb, N. G., and Rose, F. G.: Observation-Based Decomposition of Radiative Perturbations and Radiative Kernels, J. Climate, 31, 10039-10058, https://doi.org/10.1175/JCLI-D-18-0045.1, 2018.

Tornow, F., Preusker, R., Domenech, C., Carbajal Henken, C. K., Testorp, S., and Fischer, J.: Top-of-Atmosphere Shortwave Anisotropy over Liquid Clouds: Sensitivity to Clouds' Microphysical Structure and Cloud-Topped Moisture, Atmosphere, 9, 256, https://doi.org/10.3390/atmos9070256, 2018.

Tornow, F., Domenech, C., and Fischer, J.: On the Use of Geophysical Parameters for the Top-of-Atmosphere Shortwave ClearSky Radiance-to-Flux Conversion in EarthCARE, J. Atmos. Ocean. Tech., 36, 717-732, https://doi.org/10.1175/JTECH-D18-0087.1, 2019. 
van de Hulst, H.: Scaling Laws in Multiple Light Scattering under very Small Angles. (Karl Schwarzschild Lecture 1995), Rev. Mod. Astron., 9, 1-16, 1996.

Wald, L. and Monget, J. M.: Sea surface winds from sun glitter observations, J. Geophys. Res.-Oceans, 88, 2547-2555, https://doi.org/10.1029/JC088iC04p02547, 1983.

Wielicki, B. A., Barkstrom, B. R., Harrison, E. F., III, R. B. L., Smith, G. L., and Cooper, J. E.: Clouds and the Earth's Radiant Energy System (CERES): An Earth Observing System Experiment, B. Am. Meteorol. Soc., 77, 853-868, https://doi.org/10.1175/15200477(1996)077<0853:CATERE>2.0.CO;2, 1996.
Zhang, Y., Macke, A., and Albers, F.: Effect of crystal size spectrum and crystal shape on stratiform cirrus radiative forcing, Atmos. Res., 52, 59-75, https://doi.org/10.1016/S0169-8095(99)00026$5,1999$. 